\title{
Femtosecond Laser Excitation Drives Ferromagnetic Gadolinium out of Magnetic Equilibrium
}

\author{
Robert Carley, ${ }^{1,2}$ Kristian Döbrich, ${ }^{1}$ Björn Frietsch, ${ }^{1,2}$ Cornelius Gahl, ${ }^{2}$ Martin Teichmann, ${ }^{1,2}$ Olaf Schwarzkopf, ${ }^{3}$ \\ Philippe Wernet, ${ }^{3}$ and Martin Weinelt ${ }^{2}, *$ \\ ${ }^{1}$ Max-Born-Institut, Max-Born-Straße 2A, 12489 Berlin, Germany \\ ${ }^{2}$ Freie Universität Berlin, Fachbereich Physik, Arnimallee 14, 14195 Berlin, Germany \\ ${ }^{3}$ Helmholz Zentrum für Materialien und Energie (BESSY II), Albert-Einstein-Straße 15, 12489 Berlin, Germany
}

(Received 10 April 2012; published 31 July 2012)

\begin{abstract}
The temporal evolution of the exchange-split $\Delta_{2}$-like $\Sigma$ valence bands of the $4 f$-ferromagnet gadolinium after femtosecond laser excitation has been studied using angle-resolved photoelectron spectroscopy based on high-order harmonic generation. The ultrafast drop of the exchange splitting reflects the magnetic response seen in femtosecond magnetic dichroism experiments. However, while the minority valence band reacts immediately, the response of the majority counterpart is delayed by 1 picosecond and is only half as fast. These findings demonstrate that laser excitation drives the valence band structure out of magnetic equilibrium.
\end{abstract}

DOI: 10.1103/PhysRevLett.109.057401

PACS numbers: 78.20.Ls, 71.45.Gm, 73.20.At, 78.47.-p

Since its first experimental observation [1,2] optical excitation of electronic spins and ultrafast magnetization dynamics have seen intense research [3,4]. Nevertheless, details of the microscopic processes involved remain controversial and a variety of models are being explored to explain the optically-induced sub-ps (de)magnetization. These include Elliott-Yafet scattering [5], electron-electron spin-flip and exchange scattering [6-8], and superballistic electron transport [9]. Likewise the role of the spin-orbit interaction has been discussed [10-12]. Experimental signatures of magnetic dynamics have been explored in detail, elucidating the role of the laser-induced hot electron distribution and state blocking in magneto-optics and x-ray magnetic circular dichroism (XMCD) [13]. The importance of the exchange interaction has been highlighted by recent work on alloys [14], including demonstrations of deterministic ultrafast optical magnetic switching [4,15]. However, only one photoemission study has addressed changes in the exchange-split bulk band structure of ferromagnets following optical demagnetization [16].

Typically, the spin system is assumed to have a defined temperature that can be extracted from the (de)magnetization at all times following optical excitation. This assumption has been repeatedly questioned $[17,18]$ but until now not directly addressed experimentally although new magnetic phases have been observed in magnetic systems driven far from equilibrium by laser excitation $[15,19]$. Given that it takes $\approx 2$ ps to reach thermal equilibrium between the electronic system and the lattice after optical pumping, the question remains how the ultrafast and equilibrium magnetization processes compare.

The nature of its magnetic ordering (Fig. 1) makes gadolinium an ideal system in which to unravel equilibrium vs. nonequilibrium charge and spin dynamics. The half-filled $4 f$ shell leads to a large localized magnetic moment $\left(7 \mu_{B}\right)$ per atom. This is exchange-coupled to the itinerant $(5 d 6 s)^{3}$ valence electrons and polarizes their spins. These then align the moments of adjacent atoms in an indirect exchange interaction explained by the Ruderman-Kittel-KasuyaYosida (RKKY) theory, leading to magnetic ordering and exchange splitting $\left(\Delta E_{\mathrm{ex}}\right)$ of the valence bands. The thermal magnetic phase transition has already been studied by spinand angle-resolved photoelectron spectroscopy (ARPES) $[20,21]$. Time-resolved studies have established that a weak spin-lattice interaction decouples the $4 f$ spins from the lattice [22], giving a slow response time of 40 ps [23-25]. This provides a fast spin excitation channel in the valence band decoupled from slow $4 f$ spin-lattice effects.

In this Letter we perform time-resolved ARPES with femtosecond extreme-ultraviolet (XUV) pulses from highorder harmonic generation to map the transient valence band structure of $\mathrm{Gd}(0001)$ after excitation by an intense femtosecond infrared (IR) pulse. We observe that the decrease in $\Delta E_{\mathrm{ex}}$ reflects the sample magnetization but occurs in the first picosecond after laser excitation through a shift of only a minority valence band. We attribute this behavior to spin mixing in the valence bands in combination with the nearly complete alignment of the $4 f$ electron spins.

Single-crystalline, $100 \AA$ thick $\mathrm{Gd}(0001)$ films were grown epitaxially on a W(110) crystal at room temperature in a base pressure of $10^{-10} \mathrm{mbar}$ and then annealed to $650 \mathrm{~K}$. ARPES experiments were conducted at $3 \times 10^{-11}$ mbar with a view-type $100 \mathrm{~mm}$ hemispherical photoelectron analyzer. The XUV radiation was generated by focusing $775 \mathrm{~nm}, 45 \mathrm{fs}, 1 \mathrm{~mJ}$ IR pulses into 100 mbar argon. The $p$-polarized $35.6 \mathrm{eV}, 100 \mathrm{fs}$ pulses with a minimum bandwidth of $150 \mathrm{meV}$ were delivered to the sample via a toroidal grating monochromator. Tuning the $\mathrm{XUV}$ photon energy to $\approx 36 \mathrm{eV}$ probes the third Brillouin zone in the $\Gamma-M$ direction, revealing the minority and majority components of the $\Delta_{2}$-like $\Sigma$ valence band [26] (Fig. 1). In the ferromagnetic phase at $100 \mathrm{~K}$ the valence 


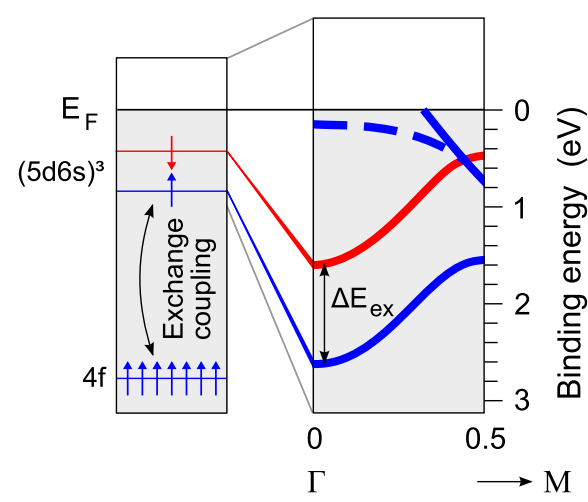

FIG. 1 (color online). Magnetic coupling (left) and calculated exchange-split valence band structure [31] of gadolinium (right). Majority spin band: blue, up arrows. Minority spin band: red, down arrows. The dashed line is the $5 d_{z^{2}}$ majority spin surface state. Bands not seen in ARPES at $36 \mathrm{eV}$ photon energy have been omitted.

band is exchange split, whereas above the Curie temperature of $293 \mathrm{~K}, \Delta E_{\mathrm{ex}}$ vanishes [20].

In the time-resolved experiments, we record ARPES images as a function of the IR pump-XUV probe delay for an absorbed pump fluence of $1.2 \mathrm{~mJ} \mathrm{~cm}^{-2}$. Pump pulses were stretched to $300 \mathrm{fs}$ to minimize space-charge distortion of the spectra. At each pump-probe delay PE spectra were obtained as shown in Fig. 2. A space-charge shift induced by the IR laser was corrected by reference to the $4 f$ peaks positions, which we have found by ARPES measurements with HeII radiation not to change between the ferro- and paramagnetic phases. The resulting binding energies are shown in Fig. 3(a). The surface state position and dynamics agree with published values [27]. At late times, when the system is in equilibrium [24], the valence band positions agree with high-resolution PE data [28].

The most striking feature of Fig. 3(a) is that within the first 2 ps the majority and minority components of the valence band exhibit different dynamical responses to the laser excitation. Specifically, the minority band reacts earlier than the majority band by $\approx 1 \mathrm{ps}$, and the rate of change of the minority binding energy is also twice as fast as its majority counterpart. To our knowledge, these distinct dynamics of the two sub-bands have not been predicted by contemporary theory, so such a large difference in reaction times was not expected. At $\approx 1$ ps the minority binding energy starts to decrease again, and by $\approx 2.5$ ps both bands attain constant binding energies until $\approx 20$ ps. Between about 30 and 60 ps we see small changes attributed to $4 f$ spin-lattice coupling, after which the binding energies correspond to those measured in thermal equilibrium [28].

Since in thermal equilibrium $\Delta E_{\text {ex }}$ is reduced with decreasing sample magnetization, it was calculated from the fitted positions of the minority and majority bands to facilite comparison with other studies and is shown in Fig. 3(b). The initial reduction in $\Delta E_{\mathrm{ex}}$ was fitted with a

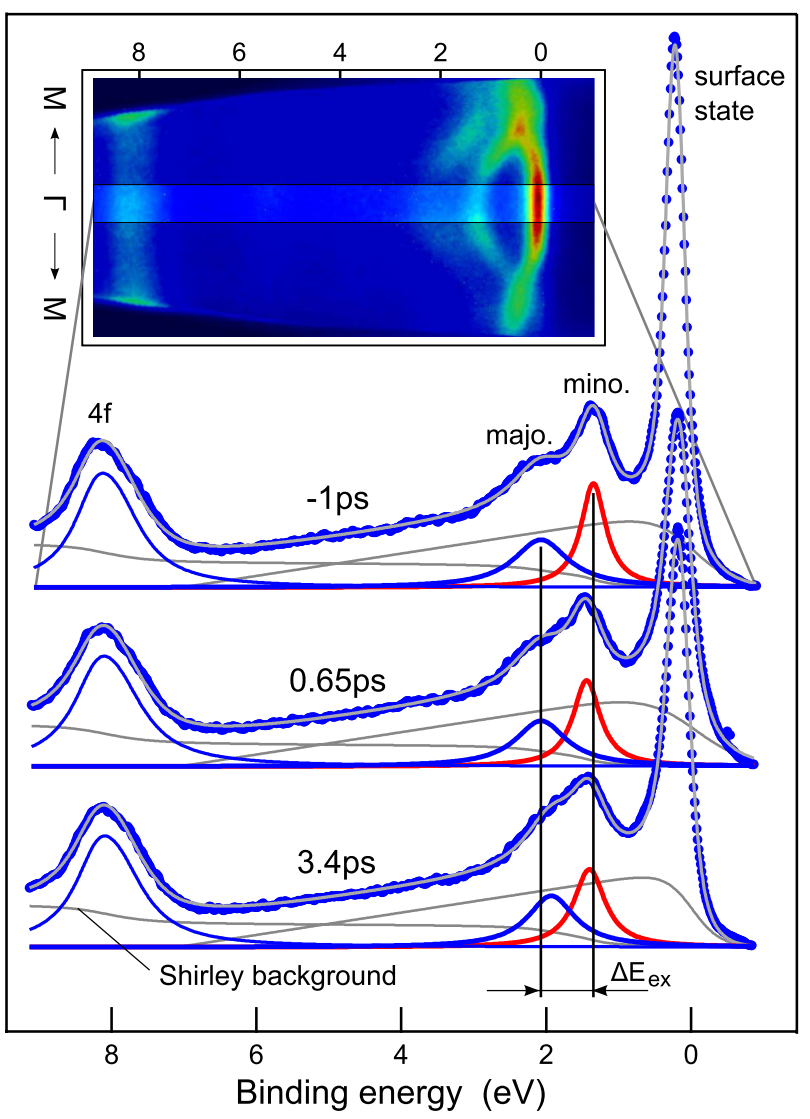

FIG. 2 (color online). Inset: ARPES of Gd at $100 \mathrm{~K}$, taken around normal emission with a photon energy of $35.6 \mathrm{eV}$. Data were integrated over $k_{\|}= \pm 0.15 \AA^{-1}$ around the $\Gamma$ point (highlighted). Main figure: PE spectra at 3 pump-probe delays fitted with the indicated spectral features. The surface state, the $4 f$ level, and the majority (blue) and minority (red) spin components of the valence band are represented as Lorentzians. A linear background accounts for elastic electron scattering from degenerate points in the Brillouin zone. Fitted spectra were convolved with a $150 \mathrm{meV}$ Gaussian instrument function dominated by the XUV spectrum.

single exponential with a time constant of $0.86 \pm 0.1 \mathrm{ps}$, in agreement with recent XMCD and magneto-optical Kerr effect (MOKE) studies [25,29]. It follows the fall in the electronic temperature [Fig. 3(b), inset axis], implying spin-flip scattering and/or transport of hot, photoexcited carriers plays a role in the demagnetization at early times. Between approximately 4 and $50 \mathrm{ps}, \Delta E_{\mathrm{ex}}$ remains constant within the experimental error. In this time window, the demagnetization rate due to spin-flip scattering has fallen with the electronic temperature. After 50 ps $\Delta E_{\text {ex }}$ recovers as the system cools.

The agreement between our $\Delta E_{\mathrm{ex}}$ data and magnetization dynamics from MOKE and XMCD show our experiment to be a complimentary tool in this field. Furthermore, our consideration of only a small region of $k$ space is justified because we see identical dynamics throughout the probed range of $k_{\|}$. Since $\Delta E_{\mathrm{ex}}$ is determined to first order by the $4 f$ 


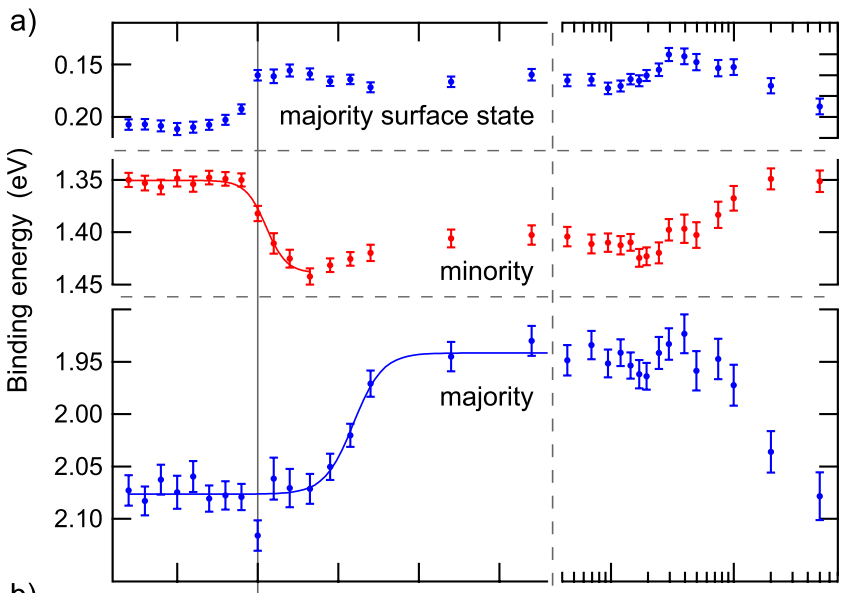

b)

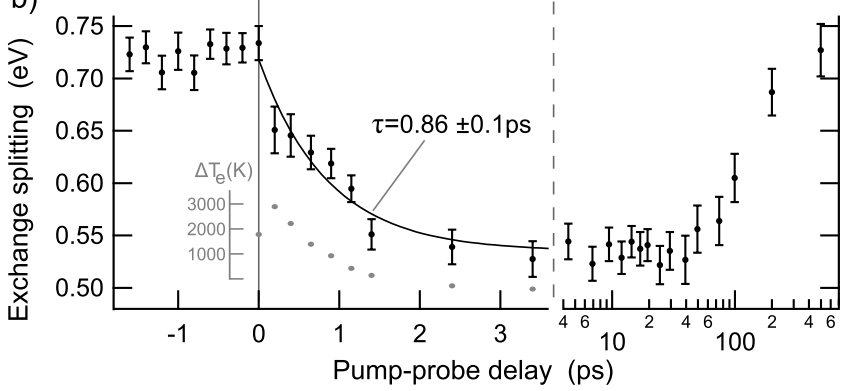

FIG. 3 (color online). (a) Binding energies of the majority and minority spin valence bands and majority surface state as a function of pump-probe delay. Solid lines are sigmoid fits to the initial changes. (b) $\Delta E_{\mathrm{ex}}$ and electron temperature as a function of pump-probe delay. Before the pump pulse the sample was at $100 \mathrm{~K}$ and the valence band $\Delta E_{\mathrm{ex}}=740 \pm 30 \mathrm{meV}$, in agreement with published data [20,21,28]. The inset axis shows the change of the electronic temperature extracted from the Fermi edge. Solid lines are exponential fits; error bars show 2 standard deviations; dashed lines mark scale changes.

spin moment and the $4 f$-valence band exchange integral [30], it lies between 0.6 and $0.9 \mathrm{eV}$ everywhere in the Brillouin zone [31,32]. We therefore expect to see similar dynamics for this band at all points in the Brillouin zone.

To rule out a thermal origin for the changes in the band structure at very short times, we plot in Fig. 4 the binding energy of the minority and majority bands against $\Delta E_{\mathrm{ex}}$. The hysteresis-like loops in Fig. 4 show that the valence band structure exhibits different behavior during laserdriven demagnetization [lower branches of the majority (blue, up triangles) and minority (red, down triangles) loops] from that during thermal remagnetization (upper branches). We note that at exactly zero pump-probe delay, a single majority data point moves to higher binding energy by $\approx 60 \mathrm{meV}$. This shift may be related to the recently observed coherent magnetic response [19] and requires investigation with better time resolution.

It is known from spin-resolved photoemission studies of gadolinium [20] that demagnetizing the sample by heating is accompanied by simultaneous reduction of both $\Delta E_{\mathrm{ex}}$ (Stoner behavior) and the spin polarization of the

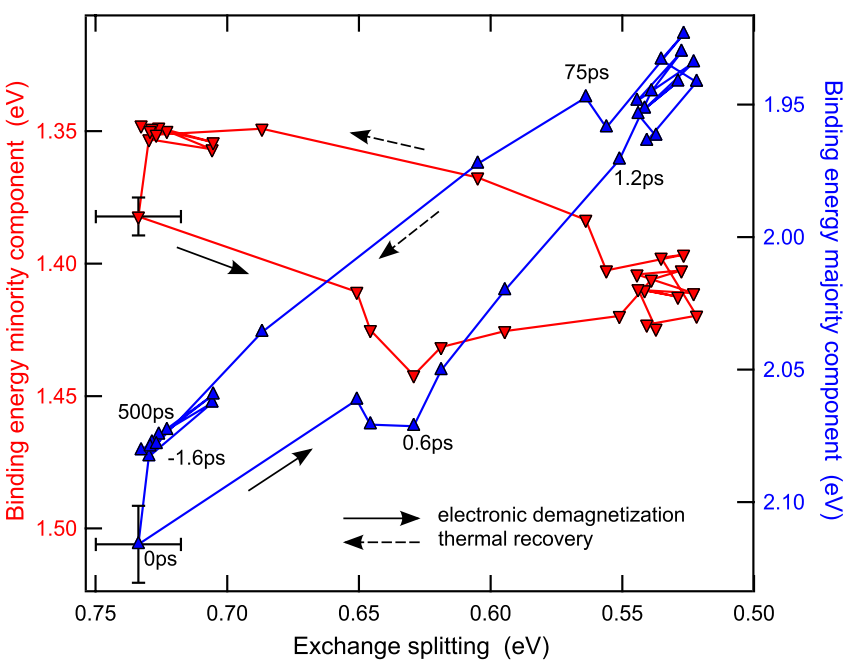

FIG. 4 (color online). Laser-driven versus thermal effects in the minority (red, down triangles, right axis) and majority (blue, up triangles, left axis) spin components of the valence band of Gd. During the first $2 \mathrm{ps}$, the system follows the lower path of each loop to smaller $\Delta E_{\mathrm{ex}}$. The electrons and lattice are then in thermal equilibrium, where they remain until the $4 f$ electrons and the lattice equilibrate at $\approx 50$ ps. Between 50 ps and $500 \mathrm{ps,}$ with the electrons, lattice, and spins all in thermal equilibrium, $\Delta E_{\mathrm{ex}}$ recovers as the system cools, moving along the upper paths to the starting position. Arrows show the direction of increasing pump-probe delay.

exchange-split bands (spin mixing). We therefore assume the changing binding energies of the bands in our data indicate a loss of spin polarization due to spin mixing. Spin mixing in the minority band is synchronous with the electronic excitation. In contrast, the majority band remains at constant binding energy and therefore does not appear to undergo spin mixing until $\approx 1$ ps later.

It might seem useful to invoke electron scattering to explain our observations. Indeed, several studies point to different spin-flip scattering rates for minority and majority electrons $[8,33,34]$. Scattering arguments, however, neglect the coupling between the valence and $4 f$ core electrons, which is artificial since they are linked by the RKKY interaction. Therefore to understand the dynamics, we consider here the coupling between the valence and $4 f$ spins shown in Fig. 1. Laser excitation with linearly polarized light cannot flip the spin of an electron and the $1.5 \mathrm{eV}$ pump energy does not directly excite electrons in the observed bands. However spin-flip processes (exchange, electron-phonon, etc.) do occur in the valence band as hot electrons scatter. When the spin of a majority valence electron is inverted, this can be transferred to the $4 f$ electron spin by the exchange interaction, leaving the majority valence band spin unchanged. This is equivalent to emitting a magnon and reduces the magnetization by $2 \mu_{B}$. The analogous spin-flip in the minority valence band, which is also exchange-coupled (albeit probably with different strength than the majority), cannot be transferred to 
the $4 f$ electrons because they are already nearly saturated with majority character [33]. The $4 f$ electrons thus act as a large majority spin reservoir, allowing the position of the majority valence band to remain unchanged for some time. At our starting temperature of $100 \mathrm{~K}$ the probability of launching a magnon will be far higher than for absorbing one [7], so we expect negligible magnon absorption at early times. Magnon absorption can explain the shift of the minority band to lower binding energy starting $\approx 1$ ps after laser excitation and implies the Gd demagnetization dynamics should depend on the starting temperature, as has indeed been observed recently [35].

In conclusion, we have seen unexpected differences in the reactions to femtosecond laser excitation of the minority and majority spin components of the valence band in ferromagnetic gadolinium. Interpretation from the perspective of spin mixing suggests that these differences arise through the majority spin saturation of the $4 f$ electrons, which favours spin-flip energy transfer from the majority valence band. It follows that, in spite of the strong exchange coupling, the minority valence band exhibits different dynamics to its majority counterpart on the subps time scale. Therefore there is no unique spin temperature on this time scale and the system is out of magnetic equilibrium. More generally, these results suggest that dynamical changes of the exchange-split band structure can considerably increase spin-flip scattering probabilites in laser-driven ultrafast demagnetization.

We thank M. Wietstruk, F. Noack, N. Zhavoronkov, R. Grosser, and R. Peslin. This work received funding from the Leibniz Gemeinschaft via Pakt für Forschung und Innovation, LASERLAB-EUROPE, and the Deutsche Forschungsgemeinschaft.

*weinelt@physik.fu-berlin.de

[1] E. Beaurepaire, J.-C. Merle, A. Daunois, and J.-Y. Bigot, Phys. Rev. Lett. 76, 4250 (1996).

[2] J. Hohlfeld, E. Matthias, R. Knorren, and K. H. Bennemann, Phys. Rev. Lett. 78, 4861 (1997).

[3] C. Stanciu, F. Hansteen, A. V. Kimel, A. Tsukamoto, A. Itoh, A. Kirilyuk, and Th. Rasing, Phys. Rev. Lett. 98, 207401 (2007).

[4] T. Ostler et al., Nature Commun. 3, 666 (2012).

[5] B. Koopmans, J. J. M. Ruigrok, F. DallaLonga, and W. J. M. de Jonge, Phys. Rev. Lett. 95, 267207 (2005).

[6] M. Krauß, T. Roth, S. Alebrand, D. Steil, M. Cinchetti, M. Aeschlimann, and H.C. Schneider, Phys. Rev. B 80, 180407 (2009).

[7] A. B. Schmidt, M. Pickel, M. Donath, P. Buczek, A. Ernst, V.P. Zhukov, P. M. Echenique, L. M. Sandratskii, E. V. Chulkov, and M. Weinelt, Phys. Rev. Lett. 105, 197401 (2010).

[8] A. Goris, K. M. Döbrich, I. Panzer, A. B. Schmidt, M. Donath, and M. Weinelt, Phys. Rev. Lett. 107, 026601 (2011).
[9] M. Battiato, K. Carva, and P. M. Oppeneer, Phys. Rev. Lett. 105, 027203 (2010).

[10] G. P. Zhang and W. Hübner, Phys. Rev. Lett. 85, 3025 (2000).

[11] C. Stamm et al., Nature Mater. 6, 740 (2007).

[12] C. Boeglin, E. Beaurepaire, V. Halté, V. López-Flores, C. Stamm, N. Pontius, H. A. Dürr, and J.-Y. Bigot, Nature (London) 465, 458 (2010).

[13] K. Carva, D. Legut, and P. Oppeneer, Europhys. Lett. 86, 57002 (2009)

[14] C. La-O-Vorakiat et al., Phys. Rev. Lett. 103, 257402 (2009).

[15] I. Radu et al., Nature (London) 472, 205 (2011).

[16] H.-S. Rhie, H. A. Dürr, and W. Eberhardt, Phys. Rev. Lett. 90, 247201 (2003).

[17] G. Zhang, W. Hübner, G. Lefkidis, Y. Bai, and T. George, Nature Phys. 5, 499 (2009).

[18] B. Koopmans, M. van Kampen, J. T. Kohlhepp, and W. J.M. de Jonge, Phys. Rev. Lett. 85, 844 (2000).

[19] J. Bigot, M. Vomir, and E. Beaurepaire, Nature Phys. 5, 515 (2009)

[20] K. Maiti, M. C. Malagoli, A. Dallmeyer, and C. Carbone, Phys. Rev. Lett. 88, 167205 (2002).

[21] K. M. Döbrich, A. Bostwick, E. Rotenberg, and G. Kaindl, Phys. Rev. B 81, 012401 (2010).

[22] S. Abdelouahed and M. Alouani, Phys. Rev. B 79, 054406 (2009).

[23] A. Vaterlaus, T. Beutler, and F. Meier, Phys. Rev. Lett. 67, 3314 (1991).

[24] A. Melnikov, H. Prima-Garcia, M. Lisowski, T. Gießel, R. Weber, R. Schmidt, C. Gahl, N. M. Bulgakova, U. Bovensiepen, and M. Weinelt, Phys. Rev. Lett. 100, 107202 (2008).

[25] M. Wietstruk, A. Melnikov, C. Stamm, T. Kachel, N. Pontius, M. Sultan, C. Gahl, M. Weinelt, H. A. Dürr, and U. Bovensiepen, Phys. Rev. Lett. 106, 127401 (2011).

[26] K. M. Döbrich, G. Bihlmayer, K. Starke, J.E. Prieto, K. Rossnagel, H. Koh, E. Rotenberg, S. Blügel, and G. Kaindl, Phys. Rev. B 76, 035123 (2007).

[27] P. A. Loukakos, M. Lisowski, G. Bihlmayer, S. Blügel, M. Wolf, and U. Bovensiepen, Phys. Rev. Lett. 98, 097401 (2007).

[28] C. Schüßler-Langeheine, Ph.D. thesis, Freie Universität Berlin, 1999.

[29] M. Sultan, A. Melnikov, and U. Bovensiepen, Phys. Status Solidi B 248, 2323 (2011).

[30] R. Ahuja, S. Auluck, B. Johansson, and M. S. S. Brooks, Phys. Rev. B 50, 5147 (1994).

[31] P. Kurz, G. Bihlmayer, and S. Blugel, J. Phys. Condens. Matter 14, 6353 (2002).

[32] K. M. Döbrich, Ph.D. thesis, Freie Universität Berlin, 2008.

[33] A. V. Fedorov, T. Valla, F. Liu, P. D. Johnson, M. Weinert, and P. B. Allen, Phys. Rev. B 65, 212409 (2002).

[34] J. Hong and D.L. Mills, Phys. Rev. B 62, 5589 (2000).

[35] M. Sultan, U. Atxitia, A. Melnikov, O. ChubykaloFesenko, and U. Bovensiepen, Phys. Rev. B 85, 184407 (2012). 